\title{
LENSING MAGNIFICATION: A NOVEL METHOD TO WEIGH HIGH-REDSHIFT CLUSTERS AND ITS APPLICATION TO SpARCS
}

\author{
H. Hildebrandt ${ }^{1,8,9}$, A. Muzzin ${ }^{2}$, T. Erben ${ }^{3}$, H. Hoekstra ${ }^{4}$, K. Kuijken ${ }^{4}$, J. Surace ${ }^{5}$, L. van Waerbeke ${ }^{1}$, \\ G. WILSON ${ }^{6}$, AND H. K. C. YeE ${ }^{7}$ \\ ${ }^{1}$ Department of Physics \& Astronomy, University of British Columbia, Vancouver, BC V6T 2C2, Canada; hendrik@phas.ubc.ca \\ ${ }^{2}$ Department of Astronomy, Yale University, New Haven, CT 06520-8101, USA \\ ${ }^{3}$ Argelander-Institut für Astronomie, Auf dem Hügel 71, 53121 Bonn, Germany \\ ${ }^{4}$ Leiden Observatory, Leiden University, Niels Bohrweg 2, 2333 CA Leiden, The Netherlands \\ ${ }^{5}$ Spitzer Science Center, California Institute of Technology 220-06, Pasadena, CA 91125, USA \\ ${ }^{6}$ Department of Physics and Astronomy, University of California-Riverside, 900 University Avenue, Riverside, CA 92521, USA \\ ${ }^{7}$ Department of Astronomy and Astrophysics, University of Toronto, 50 St George Street, Toronto, Ontario M5S 3H4, Canada \\ Received 2011 March 14; accepted 2011 April 25; published 2011 May 9
}

\begin{abstract}
We introduce a novel method to measure the masses of galaxy clusters at high redshift selected from optical and IR Spitzer data via the red-sequence technique. Lyman-break galaxies are used as a well-understood, highredshift background sample allowing mass measurements of lenses at unprecedented high redshifts using weak lensing magnification. By stacking a significant number of clusters at different redshifts with average masses of $\sim(1-3) \times 10^{14} M_{\odot}$, as estimated from their richness, we can calibrate the normalization of the mass-richness relation. With the current data set (area: $6 \mathrm{deg}^{2}$ ) we detect a magnification signal at the $>3 \sigma$ level. There is good agreement between the masses estimated from the richness of the clusters and the average masses estimated from magnification, albeit with large uncertainties. We perform tests that suggest the absence of strong systematic effects and support the robustness of the measurement. This method - when applied to larger data sets in the future-will yield an accurate calibration of the mass-observable relations at $z \gtrsim 1$ which will represent an invaluable input for cosmological studies using the galaxy cluster mass function and astrophysical studies of cluster formation. Furthermore, this method will probably be the least expensive way to measure masses of large numbers of $z>1$ clusters detected in future IR-imaging surveys.
\end{abstract}

Key words: galaxies: clusters: general - galaxies: photometry

\section{INTRODUCTION}

Galaxy clusters represent the largest collapsed structures in the universe which form in the peaks of the density field (see Voit 2005; Allen et al. 2011, for reviews). In order to relate the observed cluster population to the density peaks seen in numerical simulations, the masses of the observed clusters have to be inferred from observables. An accurate calibration of these mass-observable relations is of great importance in astrophysical studies of cluster formation and evolution as well as in cosmological studies using the galaxy cluster mass function.

The richness of a galaxy cluster (Abell 1958; Postman et al. 1996; Yee \& López-Cruz 1999; Muzzin et al. 2007a; Rozo et al. 2009) is such an observable that can easily be measured from optical/IR data out to high redshifts. The mass measurements that are required for calibration, however, become increasingly difficult at higher redshifts. The strong assumptions that are necessary to interpret X-ray (hydrostatic equilibrium) or velocity dispersion (virial equilibrium) measurements make it important to perform independent cross checks which do not rely on the same assumptions. This is particularly important at high $z$ where clusters are dynamically younger and equilibrium assumptions become increasingly questionable.

One way to measure mass that does not rely on assumptions like hydrostatic or virial equilibrium is weak gravitational

\footnotetext{
8 Also at Leiden Observatory, Leiden University, Niels Bohrweg 2, 2333 CA Leiden, The Netherlands.

9 Also at Argelander-Institut für Astronomie, Auf dem Hügel 71, 53121 Bonn, Germany.
}

lensing (WL; for a comprehensive review, see Bartelmann \& Schneider 2001). The commonly used measurement of the shearing of galaxies breaks down for higher redshifts because the sources cannot be resolved anymore and hence their ellipticities cannot be measured. This is particularly true for ground-based data and lens redshifts approaching $z \sim 1$. Those higher redshift regions are, however, of special interest for cluster astrophysics because they approach the cluster formation epoch where observational guidance is crucial. The measurements of dark energy using clusters (see, e.g., Voit 2005) as well as measurements of primordial non-Gaussianity (e.g., Mortonson et al. 2011) benefit from a large redshift baseline including high- $z$ observations.

Here we present a first measurement of the average mass of such high- $z$ cluster lenses based on the magnification effect of WL as proposed by van Waerbeke et al. (2010). This method does not require one to resolve the sources and hence can be applied at higher redshifts. By stacking a number of clusters we can measure their average mass. The clusters are selected from the Spitzer Adaptation of the Red-sequence Cluster Survey (SpARCS ${ }^{10}$; Wilson et al. 2009; Muzzin et al. 2009; Demarco et al. 2010), whereas the background sources are selected from catalogs of the Canada-France-Hawaii Telescope Lensing Survey (CFHTLenS), which are based on imaging data from the CFHT Legacy Survey (CFHTLS), via the Lymanbreak technique (Steidel et al. 1996; Giavalisco 2002). The data are presented in Section 2. In Section 3 we describe the magnification method. Results are reported in Section 4

\footnotetext{
10 http://faculty.ucr.edu/ gillianw/SpARCS
} 
and discussed in Section 5. Throughout we assume a flat $\Lambda C D M$ cosmology with $\Omega_{\mathrm{m}}=0.27, \Omega_{\Lambda}=0.73$, and $H_{0}=$ $70 \mathrm{~km}^{-1} \mathrm{~s}^{-1} \mathrm{Mpc}^{-1}$ according to Komatsu et al. (2011). All magnitudes are in the $\mathrm{AB}$ system.

\section{DATA SET}

The cluster sample used for this analysis is drawn from the $9 \mathrm{deg}^{2} X M M$-LSS field which is one of the six fields in the $42 \mathrm{deg}^{2}$ SpARCS survey. Clusters are selected as overdensities in a combined color and position space using the cluster redsequence method developed by Gladders \& Yee (2000, 2005). SpARCS uses a $z^{\prime}-3.6 \mu \mathrm{m}$ color selection which allows the detection of clusters up to redshifts as high as $z \sim 1.5$. Photometric redshifts for the clusters are calculated based on the color of the cluster red sequence and have been refined using a subsample of 33 clusters with confirmed spectroscopic redshifts. Comparison with the spectroscopic sample shows that the photometric redshifts are accurate to $\Delta z \sim 0.1$ up to $z \sim 1.4$. The richness is estimated from $3.6 \mu \mathrm{m}$ data. Since the $K-3.6 \mu \mathrm{m}$ color of galaxies is independent of star formation history and redshift for $z \lesssim 2$, these $3.6 \mu \mathrm{m}$ richness estimates are equivalent to $K$ band ones. Further details of the SpARCS cluster catalogs can be found in Muzzin et al. (2009) and Wilson et al. (2009), and a more detailed discussion of the cluster detection algorithm can be found in Muzzin et al. (2008).

In the following we concentrate on secure clusters (SpARCS flux $>9$ ). This ensures that only massive systems and no potential false positives are stacked. Selecting clusters in this way leads to more massive systems at high redshift than at low redshift, but with the limited data set used here we cannot afford more physical cuts (e.g., fixed richness). Within the overlap area between SpARCS and CFHTLS, we find 48 moderately rich and securely detected clusters with redshifts between $z=0.2$ and $z=1.6$.

As sources we use Lyman-break galaxies (LBGs) selected from catalogs of the CFHTLS field W1 which overlaps with the SpARCS $X M M$-LSS field by approximately $6 \mathrm{deg}^{2}$. The optical multi-color catalogs are created from stacked images (see Erben et al. 2009, for details on the imaging data reduction with the THELI pipeline) in a similar way as described in Hildebrandt et al. (2009a) with the notable exception that the point spread function is brought to the same Gaussian shape over the whole pointing and for all five images in the ugriz filters of each field. This allows more accurate color measurements which results in better photo- $z$ 's and a more accurate LBG selection. Details of this method will be presented in a forthcoming paper. The LBG color selection of $u-, g$-, and $r$-dropouts is identical to the one presented in Hildebrandt et al. (2009a) with the exception that $u$-dropouts are only required to have $u-g>1$ instead of $u-g>1.5$. This relaxed $u-g$ cut is identical to the one used by van der Burg et al. (2010) whose luminosity function measurements we use for calibration. ${ }^{11}$

In the following we use $z \sim 3 u$-dropouts and $z \sim 4$ $g$-dropouts. The $u$-dropouts are the cleanest background sample in terms of low- $z$ contamination and they are the lowest-redshift dropout sample detectable from the ground which results in the highest apparent brightness. The $g$-dropouts are fainter by $\sim 0.5 \mathrm{mag}$ due to their larger distance resulting in larger photometric errors. They also show larger low- $z$ contamination. Thus, we can only safely cross-correlate them to clusters with

\footnotetext{
11 This change was introduced to make the sample more comparable to other
} $u$-dropout studies, like Steidel et al. (1999). intermediate and high redshifts - beyond the redshifts of the possible contaminants. In the following, we correct for the dilution due to contamination (boosting the signal by $10 \%$ ) whenever $g$-dropouts are used. The CFHTLS-Wide data are too shallow to select $r$-dropouts in significant numbers.

\section{MASS MEASUREMENT}

The signal-to-noise ratio $(\mathrm{S} / \mathrm{N})$ of the lensing signal per background galaxy is generally lower for magnification-based methods than for shear-based ones. ${ }^{12}$ However, since magnitudes - the only requirement for magnification-are easier to measure than shapes, there are always more galaxies, and in particular higher-redshift galaxies, available for magnification. Thus, there is a break-even redshift for each data set beyond which magnification becomes more powerful than shear because ellipticity measurements become impossible. This is the case with clusters at $z \gtrsim 0.8$ where very few galaxies with reliable shape measurements are available at redshift higher than the cluster redshift, as it is very difficult to measure shapes of galaxies with $z>1$ from ground-based data.

Since the $\mathrm{S} / \mathrm{N}$ is too low for a single high- $z$ cluster to be detected via magnification with the background samples described above, we rely on stacking the signals of several clusters. In this way we can in principle estimate their average mass, a method very similar to what is usually done in galaxy-galaxy lensing. Therefore, the estimator is identical to Equation (11) in Hildebrandt et al. (2009b); i.e., we look for correlations in the positions of LBGs and the SpARCS clusters. Those positions are correlated due to the magnification-bias effect of WL.

The magnification signal scales linearly with the slope of the number counts, $\alpha .{ }^{13}$ Thus, it is important to select a source population with a large $|\alpha|$. For the interpretation of the signal, $\alpha$ needs to be measured. However, we cannot use the measured $\alpha$ directly because of incompleteness in our catalog. Furthermore, the incompleteness changes over the magnitude bin and so does the $\alpha$ itself. In order to account for these effects, we model the incompleteness as a function of magnitude by comparing the LBG number counts in the CFHTLS-Wide to the ones in the CFHTLS-Deep (Hildebrandt et al. 2009a). We then multiply the luminosity function of the LBGs measured in van der Burg et al. $(2010)^{14}$ with this incompleteness function. Next we artificially magnify the same luminosity functions for different values of the magnification $\mu$ and again multiply with the un-magnified incompleteness function. Then we compare the numbers of LBGs in a chosen magnitude bin between the un-lensed and the lensed case. A linear fit to the number excess (or depletion) as a function of $\mu$ yields $\alpha_{\text {eff }}$, the effective $\alpha$ to be used in the interpretation of the measurement.

The two problems of varying incompleteness and varying $\alpha$ over the magnitude bin should be considered in all magnification applications. Just correcting the number counts for incompleteness before measuring $\alpha$ and then averaging the individual $\alpha$ 's of all galaxies (assigned depending on their magnitudes) in the magnitude bin only works if the changes of the incompleteness

\footnotetext{
12 This depends on the slope of the number counts (see, e.g., Hildebrandt et al 2009 b). It is beneficial to select a source population with a steep slope.

13 The $\alpha$ we use here should not be confused with the faint-end slope of the luminosity function which is also often called $\alpha$.

14 Note that the volume probed by each of the dropout samples in the $4 \mathrm{deg}^{2}$ of the CFHTLS-Deep fields is $\sim 0.2 \mathrm{Gpc}^{3} h^{-1}$ so that cosmic variance on the number counts is negligible. For comparison, the Millennium Simulation has a volume of $0.125 \mathrm{Gpc}^{3} h^{-1}$.
} 

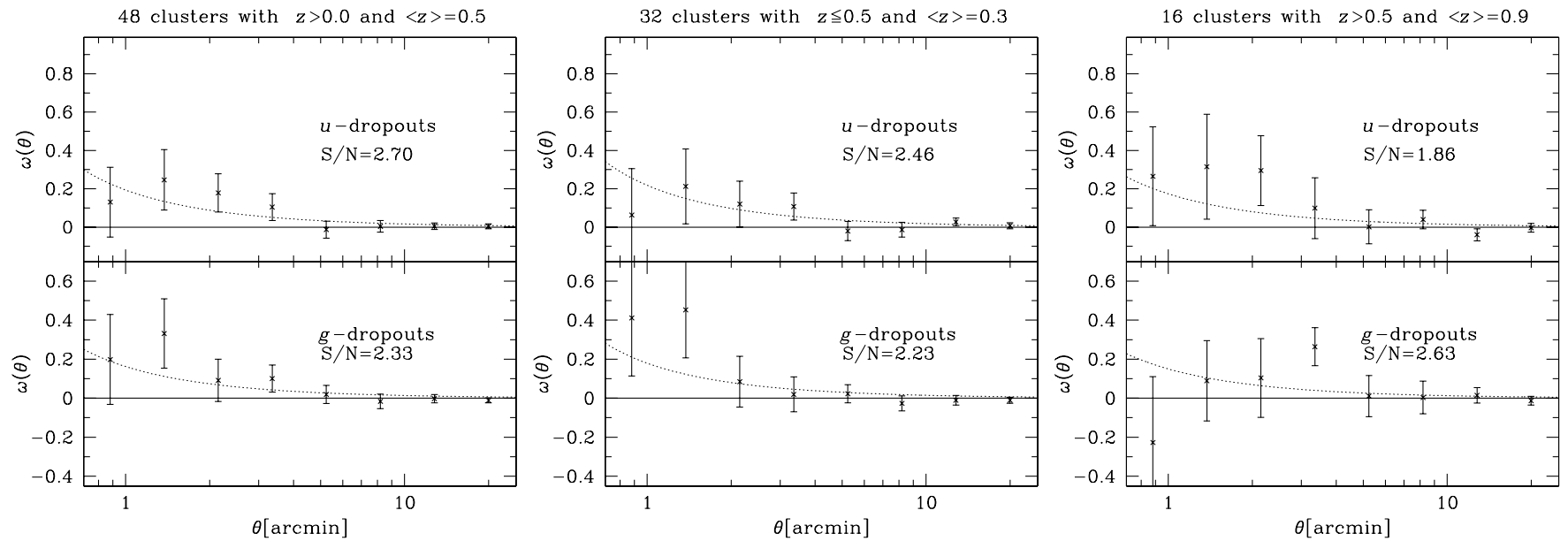

Figure 1. Magnification-induced cross-correlation between rich SpARCS cluster and $u$-dropouts (top) and $g$-dropouts (bottom) from CFHTLenS. The dotted lines correspond to the best-fit multi-SIS profiles and the $\mathrm{S} / \mathrm{N}$ values are for the individual measurements (see Table 1 for the $\mathrm{S} / \mathrm{N}$ when $u$-and $g$-dropouts are combined). Left: all 48 clusters. Middle: subsample of 32 clusters with $z \leqslant 0.5$. Right: subsample of 16 clusters with $z>0.5$.

as well as of $\alpha$ over the magnitude range are moderate. The $\alpha_{\text {eff }}$ 's that we use here can easily differ from these naive $\alpha$ estimates by $20 \%-30 \%$.

Besides estimating accurate $\alpha_{\text {eff' }}$ is is important to cleanly separate the lenses and the sources in redshift to prevent physical cross-correlations from biasing the WL measurement. Using LBGs as a background sample (and $u$-dropouts in particular) has the great advantage that they represent a very clean high- $z$ sample and their small amount of low-redshift contaminants are very well understood (Steidel et al. 1999, 2003). In this way, we can make sure that no galaxies with redshifts comparable to the cluster redshifts are included in the background samples and that physical cross-correlation (in contrast to magnification-induced cross-correlations) do not play a role here.

The cross-correlation signal $w(\theta)$ is then related to the magnification $\mu$ through (Bartelmann \& Schneider 2001)

$$
w(\theta)=\left\langle\alpha_{\mathrm{eff}}-1\right\rangle \delta \mu(\theta),
$$

with $\delta \mu(\theta)=\mu(\theta)-1$. The $\left\langle\alpha_{\text {eff }}-1\right\rangle$ for the bright LBGs selected from the CFHTLS-Wide, which we use here, is always positive, so that we expect a positive cross-correlation signal.

Since the clusters span a range of redshifts and masses-as suggested by their richness-we cannot just fit a simple cluster mass model like a singular isothermal sphere (SIS) to the stacked signal and estimate the average cluster mass directly. Rather we fit the WL signal with a one-parameter model which is constructed from a weighted sum of SIS models for all $N$ clusters:

$$
\mu_{\mathrm{comb}}(a)=\sum_{i=0}^{N} \mu_{\mathrm{SIS}}\left(z_{i} ; a M_{200, \text { richness }, i}\right) .
$$

The relative weighting between the clusters in this model takes the different redshifts $z_{i}$ (and hence different lensing efficiencies and different critical densities) as well as the

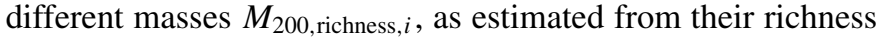
via Equation (9) of Muzzin et al. (2007b), into account. Hence, the fit does not directly yield an average mass. Instead the normalization of the mass-richness relation is fitted under the assumptions that the slope of this relation does not change with redshift, the redshifts for the clusters are accurate, and that the background cosmology is known.
The magnification for an SIS takes the form

$$
\mu(\theta)=\frac{\theta}{\theta-\theta_{\mathrm{E}}},
$$

with $\theta_{\mathrm{E}}=4 \pi\left(\frac{\sigma_{v}}{c}\right)^{2} \frac{D_{\mathrm{ds}}}{D_{\mathrm{s}}}$ being the Einstein radius of an SIS with velocity dispersion $\sigma_{v} ; D_{\mathrm{s}}$ and $D_{\mathrm{ds}}$ are the angular diameter distances from observer to source and from deflector to source, respectively. The velocity dispersion is related to the mass, $M_{200}$, by

$$
\sigma_{v}^{3}=\frac{1}{2^{3 / 2}} M_{200} \sqrt{\frac{4}{3} \pi 200 \rho_{\text {crit }}(z) G^{3}},
$$

where $\rho_{\text {crit }}(z)$ is the critical density at the cluster redshift, $z$, and $G$ is the gravitational constant. Here we estimate the masses with the mass-richness relation at $z \sim 0.3$ from Muzzin et al. (2007b) which is based on dynamical mass estimates. A fitted $a$ of unity would mean that the mass-richness relation at the redshifts of our cluster is identical to the one from Muzzin et al. (2007b). The mass estimated from magnification becomes $M_{\text {magnification }}=a M_{\text {richness }}$.

In the future, larger cluster samples will allow us to bin the clusters much more finely so that an equal weighting can be used and no external mass-observable relation is needed for proper scaling.

\section{RESULTS}

Figure 1 shows the cross-correlation signal between rich SpARCS clusters in two different redshift bins and the $u$-dropouts with $23<r<24.5\left(\left\langle\alpha_{\text {eff }}-1\right\rangle=1.54\right.$; surface density $\left.0.26 \mathrm{arcmin}^{-2}\right)$ and $g$-dropouts with $23.5<i<25$ $\left(\left\langle\alpha_{\text {eff }}-1\right\rangle=1.24\right.$; surface density $\left.0.24 \operatorname{arcmin}^{-2}\right) .{ }^{15}$ The mean masses of the clusters are estimated from their richness. Errors on the data points represent the standard deviation between the signals for the different clusters and thus reflect Poissonian noise as well as noise due to clustering of the background population and noise introduced by structures along the lines of sight to the clusters (Hoekstra et al. 2011). The off-diagonal elements

\footnotetext{
15 When comparing surface densities between shear and magnification to estimate the $\mathrm{S} / \mathrm{N}$, the magnification densities should be scaled by a factor $\left(2\left|\alpha_{\text {eff }}-1\right| \sigma_{\epsilon}\right)^{2}$ (Schneider et al. 2000), with $\sigma_{\epsilon}$ being the intrinsic ellipticity dispersion.
} 
Table 1

Results of the Multi-SIS Fits to the Magnification Signals

\begin{tabular}{lcccccc}
\hline \hline Sample & Number of Clusters & $\langle z\rangle$ & $\begin{array}{c}M_{\text {richness }} \\
\left(\times 10^{14} M_{\odot}\right)\end{array}$ & S/N & $a$ & $\begin{array}{c}M_{\text {magn. }} \\
\left(\times 10^{14} M_{\odot}\right)\end{array}$ \\
\hline All & 48 & 0.5 & 1.9 & 3.6 & $1.1_{-0.6}^{+0.6}$ & $2.1_{-1.1}^{+1.2}$ \\
$z \leqslant 0.5$ & 32 & 0.3 & 1.1 & 3.3 & $1.4_{-0.7}^{+0.8}$ & $1.5_{-0.8}^{+0.9}$ \\
$z>0.5$ & 16 & 0.9 & 3.4 & 3.2 & $0.9_{-0.7}^{+0.9}$ & $3.1_{-2.5}^{+3.0}$ \\
\hline
\end{tabular}

of the covariance matrix are estimated by measuring the crosscorrelation signal between 1000 fake clusters placed at random positions and the background LBGs.

We fit Multi-SIS (see Equation (2)) profiles to the combined $u$ - and $g$-dropout signals for the two cluster samples in the range $0{ }^{\prime} 8<\theta<20^{\prime}$. Table 1 summarizes the results. The WL signal is - within the large errors-consistent with the signal expected from the Muzzin et al. (2007b) mass-richness relation, as indicated by the values for $a$ being consistent with unity. Despite similar $\mathrm{S} / \mathrm{N}$ the relative error of the mass is larger for the high- $z$ sample than for the low $-z$ sample. That is due to the fact that the high- $z$ g-dropout signal is not very well fit by the multi-SIS profile (but still consistent within noise). Note that we just used the scaling with richness to construct the multi-SIS function and not the absolute normalization of the mass-richness relation. Separate multi-SIS fits to the signals of the $u$ - and $g$-dropouts of a given cluster sample yield consistent results as well. In the following, we perform two tests for systematic errors that could affect our result.

The SpARCS $X M M$-LSS field also overlaps with the CFHTLS-Deep field D1. In this $1 \mathrm{deg}^{2}$ field, much deeper imaging is available which allows the selection of fainter LBGs (the ones also used in Hildebrandt et al. 2009a, 2009b) which have negative $\left\langle\alpha_{\text {eff }}-1\right\rangle$. We find eight of the 48 clusters in that field. Although the statistical power is limited due to the small number of clusters and the lower absolute value of $\left\langle\alpha_{\text {eff }}-1\right\rangle$ (somewhat compensated by the higher LBG density), these faint LBGs allow us to perform an important test. While the positive cross-correlations of the brighter LBGs could in principle also be caused by improper redshift separation and hence physical cross-correlations, anti-correlations can only be caused by WL. Here we use faint $u$ - and $g$-dropouts with $r>25.8$ and $i>26.3$, respectively, which correspond to parts of the LBG luminosity function where $\left\langle\alpha_{\text {eff }}-1\right\rangle=-0.09$ and $\left\langle\alpha_{\text {eff }}-1\right\rangle=-0.18$. Their surface densities are $2.7 \mathrm{arcmin}^{-2}$ and $0.83 \mathrm{arcmin}^{-2}$.

As expected for a background sample with $\left\langle\alpha_{\text {eff }}-1\right\rangle<0$, we find under-densities of LBGs around the clusters. The detection is less significant due to the limited number of clusters and the lower absolute values of $\left\langle\alpha_{\text {eff }}-1\right\rangle$ which cannot be compensated by the higher surface density of dropouts in the CFHTLS-Deep and their lower intrinsic clustering signal. ${ }^{16}$

Another useful test is to cross-correlate the clusters to a population of objects that should not show any correlation in their positions because it is closer to the observer than the clusters. For that test, we select stars and measure their cross-correlation function to the clusters. We do not detect any significant cross-correlation signal for stellar samples of

\footnotetext{
16 The main noise term in the magnification measurement is not the Poissonian shot noise, which originates from the finite sampling of the $\kappa$-field with a limited number of background objects, but the noise introduced by the intrinsic clustering of the background galaxies (see van Waerbeke 2010, for a full treatment of the different error terms). As we showed in Hildebrandt et al. (2007, 2009a) the clustering of the faint LBGs is much weaker than the clustering of the bright LBGs.
}

different limiting magnitudes. It should be noted that the stellar auto-correlation function is different from zero (with a very low amplitude, but high significance) so that a non-detection of a cross-correlation signal between stars and clusters is a meaningful test.

From these two tests we conclude that our measurement is robust and free of strong systematic biases. However, the current sample is too small to study more subtle systematic effects in detail.

\section{DISCUSSION AND OUTLOOK}

We measure the normalization of the high-redshift mass-richness relation of galaxy clusters selected from SpARCS employing a novel method based on the WL magnification effect of these clusters on $z \gtrsim 3$ LBGs. Even with a small sample of rich, high- $z$ clusters and optical data of modest depths, we detect a magnification signal at $>3 \sigma$ significance which yields a measurement of this normalization (equivalent to a measurement of the average cluster mass) with a relative error of $\sim 50 \% .{ }^{17}$ The measured normalization is in good agreement with the low- $z$ mass-richness relation from Muzzin et al. (2007b) which is based on dynamical mass estimates.

Using much deeper data on a $1 \mathrm{deg}^{2}$ sub-field, we detect an anti-correlation between a few high- $z$ SpARCS clusters and faint LBGs, supporting a WL origin of the signal and suggesting that the measurement on the shallower data is free of physical cross-correlations that could mimic a WL signal.

The overlap area between SpARCS and CFHTLS is too small to subdivide the clusters into different richness bins and estimate a full mass-richness relation at high redshift. However, it is well within reach of current facilities to expand this area by an order of magnitude through either observing IR, X-ray, or Sunyaev-Zel'dovich (SZ) surveys with ground-based optical imaging or through targeting optical surveys such as the CFHTLS with IR or X-ray imaging from space or with SZ telescopes.

We would like to stress that this method represents a way to obtain mass estimates of large samples of clusters at very high redshifts that is relatively cheap in terms of telescope time. It is the only method that can realistically be applied to measure the masses of very large numbers of $z>1$ clusters to be found by future high- $z$ cluster surveys. Concentrating on the bright part of the LBG luminosity function and probably also on the lowest redshift and hence brightest LBGs reachable from the ground ( $u$-dropouts) seems the most promising route. This certainly requires high-quality $u$-band imaging, which is available at only a few facilities.

Our study is based on observations obtained with MegaPrime/MegaCam, a joint project of CFHT and CEA/ DAPNIA, at the CFHT which is operated by the NRC of Canada, the CNRS of France, and the University of Hawaii and on observations made with the Spitzer Space Telescope, which is operated by the Jet Propulsion Laboratory, California Institute of Technology, under a contract with NASA. This work is based in part on data products produced at TERAPIX and CADC.

We thank the CFHTLenS team for their work on the CFHTLenS data products which are used in this study. $\mathrm{H}$. Hildebrandt is supported by the Marie Curie IOF 252760 and by a CITA National Fellowship. T.E. is supported by the BMBF

\footnotetext{
17 Note that the relative error of $a$ scales as $\left(\frac{S}{N}\right)^{-2 / 3}$ for data that agree within
} errors with the model. 
through project "GAVO III" and by the DFG through project ER 327/3-1 and the TR 33. H. Hoekstra acknowledges support from the NWO and a Marie Curie IRG. L.v.W. is supported by NSERC and CIfAR. G.W. acknowledges support from NSF grant AST-0909198.

Facilities: Spitzer, CFHT

\section{REFERENCES}

Abell, G. O. 1958, ApJS, 3, 211

Allen, S. W., Evrard, A. E., \& Mantz, A. B. 2011, ARA\&A, in press (arXiv:1103.4829)

Bartelmann, M., \& Schneider, P. 2001, Phys. Rep., 340, 291

Demarco, R., et al. 2010, ApJ, 711, 1185

Erben, T., et al. 2009, A\&A, 493, 1197

Giavalisco, M. 2002, ARA\&A, 40, 579

Gladders, M. D., \& Yee, H. K. C. 2000, AJ, 120, 2148

Gladders, M. D., \& Yee, H. K. C. 2005, ApJS, 157, 1

Hildebrandt, H., Pielorz, J., Erben, T., Schneider, P., Eifler, T., Simon, P., \& Dietrich, J. P. 2007, A\&A, 462, 865

Hildebrandt, H., Pielorz, J., Erben, T., van Waerbeke, L., Simon, P., \& Capak, P. 2009a, A\&A, 498, 725

Hildebrandt, H., van Waerbeke, L., \& Erben, T. 2009b, A\&A, 507, 683

Hoekstra, H., Hartlap, J., Hilbert, S., \& van Uitert, E. 2011, MNRAS, 412, 2095
Komatsu, E., et al. 2011, ApJS, 192, 18

Mortonson, M. J., Hu, W., \& Huterer, D. 2011, Phys. Rev. D, 83, 023015

Muzzin, A., Wilson, G., Lacy, M., Yee, H. K. C., \& Stanford, S. A. 2008, ApJ, 686, 966

Muzzin, A., Yee, H. K. C., Hall, P. B., Ellingson, E., \& Lin, H. 2007a, ApJ, 659, 1106

Muzzin, A., Yee, H. K. C., Hall, P. B., \& Lin, H. 2007b, ApJ, 663, 150

Muzzin, A., et al. 2009, ApJ, 698, 1934

Postman, M., Lubin, L. M., Gunn, J. E., Oke, J. B., Hoessel, J. G., Schneider, D. P., \& Christensen, J. A. 1996, AJ, 111, 615

Rozo, E., et al. 2009, ApJ, 703, 601

Schneider, P., King, L., \& Erben, T. 2000, A\&A, 353, 41

Steidel, C. C., Adelberger, K. L., Giavalisco, M., Dickinson, M., \& Pettini, M. 1999, ApJ, 519, 1

Steidel, C. C., Adelberger, K. L., Shapley, A. E., Pettini, M., Dickinson, M., \& Giavalisco, M. 2003, ApJ, 592, 728

Steidel, C. C., Giavalisco, M., Pettini, M., Dickinson, M., \& Adelberger, K. L. 1996, ApJ, 462, L17

van der Burg, R. F. J., Hildebrandt, H., \& Erben, T. 2010, A\&A, 523, A74

van Waerbeke, L. 2010, MNRAS, 401, 2093

van Waerbeke, L., Hildebrandt, H., Ford, J., \& Milkeraitis, M. 2010, ApJ, 723, L13

Voit, G. M. 2005, Rev. Mod. Phys., 77, 207

Wilson, G., et al. 2009, ApJ, 698, 1943

Yee, H. K. C., \& López-Cruz, O. 1999, AJ, 117, 1985 